\title{
Assessing Poggendorff effects via collinearity, perpendicularity, parallelism, and Oppel (distance) experiments*
}

\author{
DANIEL J. WEINTRAUB $†$ and LILLIAN TONG \\ Human Performance Center, University of Michigan, Ann Arbor, Michigan 48104
}

\begin{abstract}
By adjusting the orientation of, and separation between, two free-standing dots, Ss indicated directions and distances associated with the Poggendorff display (a transversal interrupted by parallel lines). Judged distance between parallels (with transversal absent) increased slightly when additional interior parallels were added; this Oppel effect can be interpreted as contour repulsion. Errors in judging the orientation of an actual transversal segment were too small to account for the Poggendorff effect. The usual large errors occurred for estimates of the orientation of the missing transversal segment between the parallel lines. Cognitive mistracking adequately describes the Poggendorff effect. Mistracking is a function of the angle stibtended between transversal and parallels, and of the orientation of the entire display.
\end{abstract}

The traditional Poggendorff display consists of two parallel lines interrupting a transversal; the transversal segments do not appear collinear. Contour-interaction theories claim that perception is distorted by the displacement of the neural representation of the contours forming the physical stimulus. For example, the Poggendorff effect is logically explained by postulating that parallels appear too close, i.e., through lateral interactions, neural contours attract one another (discussed by Tong \& Weintraub, 1974). Alternatively, the acute angle between transversal and parallels might appear too large, an instance of contour repulsion (Blakemore, Carpenter, \& Georgeson, 1970; Burns \& Pritchard, 1971).

The initial experiments are direct offshoots of our previous work (Tong \& Weintraub, 1974), attempting to assess the parallels-attract hypothesis applied to the Poggendorff effect. If parallels between transversal segments are represented in experience as too close together, then the attached transversals will seem to be vertically misaligned when they are actually collinear. Additional parallel lines were added between the original parallels under the assumption that the additional parallels would cause increasing contour attraction and therefore an increasing Poggendorff effect. However, the data clearly showed that extra interior parallels reduced the Poggendorff error (Tong \& Weintraub, 1974). The Oppel effect (Robinson, 1972), the overestimation of filled space

* Support was provided by a United States Publich Health Service Research Scientist Development Award (K2-MH-35,253) to D. J. Weintraub. Research funds were provided by National Science Foundation Grant GB8181.

†Address: Human Performance Center, Perry Building, 330 Packard Road, Ann Arbor, Michigan 48104. relative to unfilled space, seemed able to account for the findings. The Poggendorff display containing extra parallels is directly analogous to an Oppel display containing many interior line segments parallel to end segments defining an interval. The first two experiments continue the investigation of the role of the parallels.

\section{EXPERIMENT I}

\section{Method}

The paid Ss were 40 students at the University of Michigan, tested one at a time. Each $S$ received eight white stimulus sheets, $21.6 \times 27.9 \mathrm{~cm}(81 / 2 \times 11 \mathrm{in}$.) containing Multilithed black lines and dots. Vertical lines, $.4 \mathrm{~mm}$ wide, were drawn parallel to the longer edges of a sheet. The separation between outer parallels was either 32 or $42 \frac{2}{3} \mathrm{~mm}$. (These choices were dictated by the separations employed in the Tong \& Weintraub, 1974, experiments.) At each of the two separations, $2,3,4$, or 5 evenly spaced parallel lines were drawn. A dot on the left parallel and a dot on the right parallel defined a direction through the field of parallels at an angle of $+45 \mathrm{deg}$ from horizontal. Each dot was black and $1.5 \mathrm{~mm}$ in diam. The 5-line stimulus display is depicted in Fig. 1.

Procedure. The $S$ sat at a desk with the eight stimuli face down in a randomized pile to his left. He was instructed to turn the top sheet face up in front of himself, with the short $(81 / 2$ in.) edge flush with the front edge of the desk. In this position, the parallel lines proceeded away from $S$. With the pencil provided, $S$ was instructed to place a small dot on an extension of the imaginary line defined by the two dots lying on the parallels, the same distance from the right dot as the right dot was from the left dot. Stated differently, the $S$ was asked to place a third dot to the right of the two dots on the sheet, so that the three dots were collinear and the S's dot was the same distarice from the middle dot as the left dot was from the middle dot. The instructions were illustrated by referring to a sample stimulus sheet. The $S$ was encouraged to erase and reposition his dot until he was satisfied. The $S$ circled his final judgment (to avoid any scoring ambiguity), turned the sheet over in a pile to his right and proceeded to the next sheet. Head tilting, drawing lines, etc., were not permitted. Subjects were allowed only to view the display and position a dot. Eye-to-stimulus distance was 


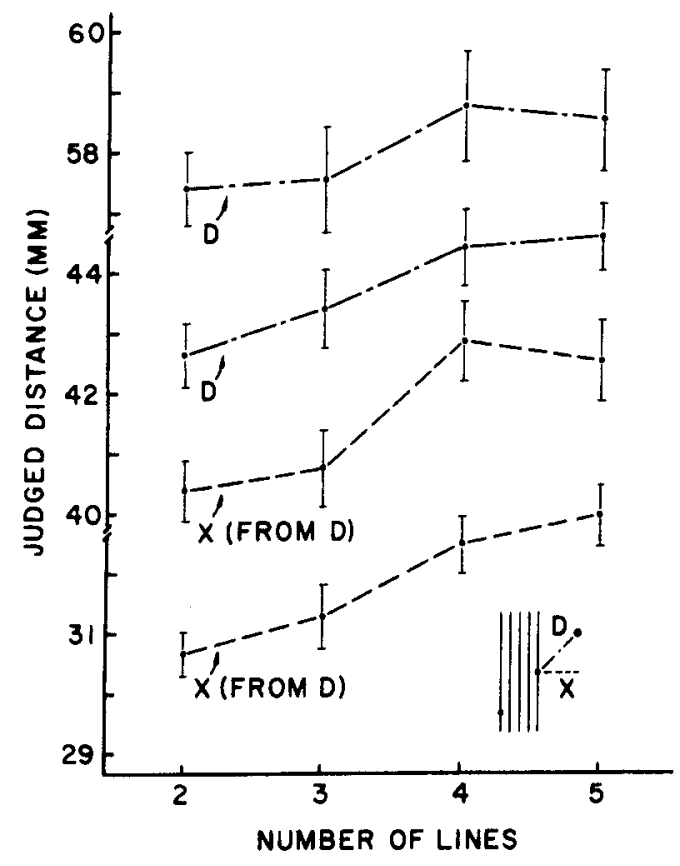

Fig. 1. Mean distance judgments for Experiment $I$. $( \pm 1$ standard error of the mean is shown by vertical bars about the mean. The true distances associated with each function are, from top to bottom, 60.34, 45.25, 42 $2 / 3$, and $32 \mathrm{~mm}$.) $A$ stimulus display is depicted at the lower right; the dot farthest to the right represents S's Judgment.

approximately $40 \mathrm{~cm}$. Angle of regard approximated $80 \mathrm{deg}$ from vertical.

\section{Results and Discussion}

In Fig. 1, note that, for either separation between parallels, mean judged slant distance, labeled $D$, increased as more lines were added. On all figures, vertical bars about each mean depict \pm 1 standard error of the mean $\left(\mathrm{s}_{\overline{\mathrm{x}}}\right)$. [At $a=.01$, the number of lines produced a statistically significant main effect, $F(3,117)=4.16$; it was obvious that the objective distance between outer parallels would do likewise, $F(1,39)=1,041.98$. The interaction was not significant.] An indirect estimate of the perceived distance between parallels can be derived from the slant distance between dots by taking the horizontal (X) component of a judgment. These mean data are plotted in the lower half of Fig. 1, and they also increased with an increase in the number of parallel lines. Subjects might have produced the increasing slant distance by increasing the slope of their collinearity judgments while holding the horizontal component constant. However, the data shown in Fig. 1 support the interpretation that adding interior parallels increased perceived distance between the outer parallels, of which $X$ is an indirect measure derived from the judged slant distance, $D$.

Adding interior lines reduced the perceived angle of inclination as shown by the lower functions in Fig. 2. The measure plotted is the mean slope of the line between the dot on the right parallel and the free-standing dot representing S's judgment. A veridical judgment would provide a slope of 1.0 (tan $45 \mathrm{deg}=1.0$ ). For a two-line display, neither function is significantly different from a tangent of 1.0 , but both functions decreased as interior parallels were added. [For these tangent data at the bottom of Fig. 2, with an .01 level test, number of lines was significant, $F(3,117)=10.15$; objective distance between outer parallels was significant, $F(3,117)=$ 10.15; objective distance between outer parallels was significant, $F(1,39)=11.04$; the interaction was not.] The outcome is consistent with the hypothesis that filled space is perceived as more extensive than unfilled space. If the dots lying on the parallels are perceived as farther apart, then the slope of the imaginary line between them should decrease.

The disturbing aspect of the outcome is that the mean distance judgments shown in Fig. 1 are primarily underestimates. In what sense can filled space be considered overestimated when the obtained distance judgments are underestimates? At this juncture, two attributes of the stimulus displays became obvious to us. First, the unfilled space was directly adjacent to the filled space, and the additional parallels used to fill the space might well have influenced the unfilled region from which the measurements were obtained. (The same criticism can be leveled at any study of the Oppel effect employing adjacent regions.) Second, the stimulus display could be considered a degraded variation of the Poggendorff display in which segments of the transversal had been replaced by dots. This particular variant has been shown to give a small Poggendorff effect (Krantz \&

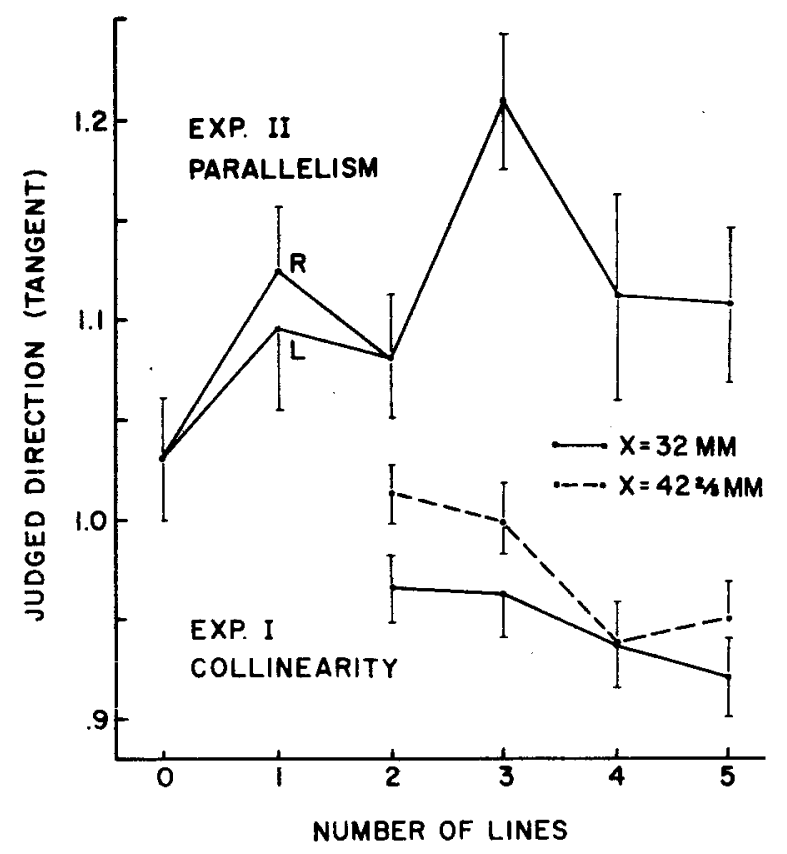

Fig. 2. Mean directional judgments for Experiments I and II (true direction is $45 \mathrm{deg}, \tan =1.0$ ). 
Weintraub, 1973, Stimulus 4, Experiment VII). An alternative to a collinearity judgment is a parallelism judgment. The stimulus used as an assessment device can be removed from the region where perturbing influences are presumed to be operating.

\section{EXPERIMENT II}

\section{Method}

A new sample of 40 paid Ss, students at the University of Michigan, was tested. Multilithed stimulus sheets, like those of Experiment I, contained dots and vertically oriented parallel lines. A stimulus sheet always contained a pair of dots (each $1.5 \mathrm{~mm}$ in diam) lying either horizontally with respect to one another, or obliquely, i.e., tilted at $+45 \mathrm{deg}$ from horizontal. The horizontal distance, $X$, between a pair of dots was fixed at $32 \mathrm{~mm}$. Vertical parallel lines $(.4 \mathrm{~mm}$ thickness) were drawn in the space between the pair of dots as well as through the dots. A given display contained from 0 through 5 vertical lines. The 0 -line display contained only the dot pair. There were two different 1-line displays, one with a vertical line through the left dot, the other with a vertical line through the right dot. The 2 -line display contained a line through each dot. Displays with 3 or more lines contained evenly spaced lines added to the interior of the 2-line display. Each of the 14 displays appeared to the left of center on a stimulus sheet. Included on every sheet was a $1.5-\mathrm{mm}$ black dot placed randomly to the right of the display with the restriction that it not be at the same height as either dot on the left. (The diagram in Fig. 3 depicts two dots on the right parallel, but only one appeared in any given display.)

Procedure. Experimental procedures were like those in the first study except that $\mathbf{S}$ was now asked for a judgment of parallelism. Again, head tilting was not allowed. He was told to observe the distance and direction between the pair of dots on the left, and to complete a pair on his right to match in distance and direction. The single dot (to S's right) represented the left dot of the pair to be completed. The instructions were: "Look at the two dots to the left, then look to the right, and try to recreate the relationship."

\section{Results and Discussion}

The function labeled $X$ in Fig. 3 represents the mean judged distance between a pair of horizontal dots. With no lines present, the true width between dots of $32 \mathrm{~mm}$ was judged as essentially $32 \mathrm{~mm}$. Adding a line through either the left or right dot decreased judged distance. A line through both dots led to a further decrease. [As an illustration of statistical significance, the differences among distance judgments for displays of 0,1 (left), and 2 lines for the $X$ function of Fig. 3 produced $F(2,78)=$ 19.11 , an outcome well beyond the .01 -level criterion.] The addition of one or more interior lines (designated as 3, 4, and 5 lines in Fig. 3) produced a statistically significant $(a=.01)$ increase in judged distance over 2 lines, which are the outer parallels alone. For judgments of slant distance, the function labeled $D$, adding parallels through the dots again caused judgments to decrease significantly $(a=.01)$. However, there was no rise with the addition of interior lines.

These data resolve the dilemma posed by the first experiment that nearly all distances were underestimated. The displays of Experiment I can be considered hybrids. Let us logically separate their components in order to facilitate a comparison with Experiment II. In Experiment I (Fig. 1), the portion of the display requiring $S$ 's attention during the dot-setting aspect of the judging (the comparisonstimulus portion so to speak) is bounded by a dot lying on the right parallel and a dot not on a line, the dot produced by S. In Experiment II (Fig. 3), the comparison stimulus is composed of two unattached dots, one produced by $S$. Therefore, the comparisonstimulus conditions differ between experiments. But a link exists. The comparison-stimulus conditions of Experiment I are duplicated by a 1-line display in Experiment II. Thus, the line-and-a-dot comparisonstimulus configuration of Experiment I can be evaluated in Experiment II with respect to the two-dot configuration. Relative to two free-standing dots, the distance between a pair of dots is underestimated when one lies on a line, and the underestimation is more pronounced when each dot of a pair lies on a line (as can be seen in Fig. 3, solid-line functions). One can account for Experiment I in terms of the outcome of Experiment II. In Experiment I, S set his free-standing dot closer to the right parallel because, when both distances were objectively equal, the distance between the dots lying on the parallels seemed smaller than the distance between the dot on the right parallel and a free-standing dot.

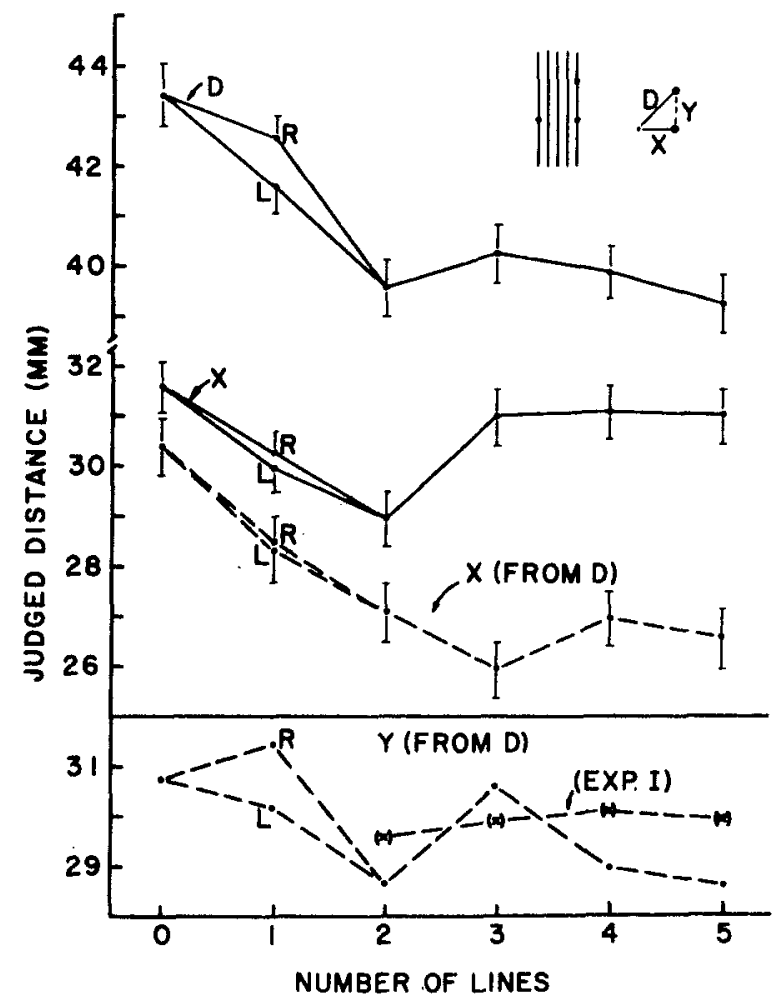

Fig. 3. Mean distance judgments for Experiment II, with a stimulus display depicted at the upper right. (The true slant distance, $D$, is $45.25 \mathrm{~mm}$; distances $X$ and $Y$ are $32 \mathrm{~mm}$ ). Derlved vertical distances, $Y$, are also shown for Experiment $I$. 
With the addition of interior lines in Experiment I, judged distances increased (i.e., underestimates decreased), the usual Oppel effect. For a similar Oppel effect in Experiment II, the additional interior lines should have produced a consistent increase in judged distance relative to two lines. For a horizontal distance, $X$, the judgments did increase when the third line was added. For slant distance, $D$, there was no change with the addition of interior lines. Is the Oppel effect most salient when adjoining distances are being judged as in Experiment I? Overall, the ovidence from both experiments plus the TongWeintraub (1974) data point toward an Oppel effect: Filled space appears more extensive than unfilled space. The contours that bound the distances being judged cannot be ignored because they exert a considerable influence on the outcome.

The derived functions, $X$ (from D) and $Y$ (from D), in Fig. 3 represent the $X, Y$ coordinates of the judged slant distance, $D$. Derived $X$ is everywhere less than $X$ judged directly. Adding lines produced a greater change in derived $X$ than in derived $Y$. Therefore, Ss can be presumed to vary their slope judgments mainly by varying dot displacement horizontally.

Judged slopes for Experiment II are shown in Fig. 2. The dominant finding is that the slope between dots of 1.0 is significantly overestimated whenever any lines are present in the display. (Note, in Fig. 2, that these particular means lie more than $1.96 \mathrm{SDs}$ above the objective value of 1.0.) The result is not easy to interpret. The fact that it is in the opposite direction from the outcome of Experiment $I$ is not surprising, because collinearity judgments were linked to the perceived distance between dots being judged, while the parallelism judgments utilized in Experiment II were not. We had anticipated that judged slopes would rise as the number of lines increased from 0 to 2 , reflecting an increasing slope with decreasing perceived distance, and then decrease slowly out to the 5-line display, reflecting the Oppel effect. The data from the 2-line display are clearly too discrepant to support such a hypothesis. An important finding concerning slope estimations for dots lying on parallels is that the slope errors (overestimations) lie in the opposite direction from errors that could lead to a Poggendorff effect.

In summary, the evidence from the first two experiments leads to several important conclusions. If objective distances are equal, then the perceived distance between a free-standing dot pair is greatest, followed by a dot pair of one free dot and one dot on a line, followed by a dot pair with each dot on a line. With respect to the Oppel effect itself, the marks used to delineate the boundaries of distances being compared should be identical, in order not to introduce the confounding effects of differential boundary markings. The Oppel effect is evidently not large under the experimental conditions employed, but the data taken in the aggregate do imply that adding interior parallels increased the judged distance between outer parallels. Slopes between two dots at a 45-deg angle and lying on parallels were shown to be overestimated.

In what sense can measurements of the reference stimulus, free-standing dots, be said to "measure" perceived orientation or perceived distance? The implied preface to every statement about data is "relative to the manner in which the free-standing dots are perceived." The proviso is not merely a technical nicety. For example, do parallels attract? The questions cannot be answered directly. Relative to two free-standing dots, parallels are perceived as closer together. But free-standing dots might repel one another, and parallels might also repel, but less strongly. From the converging evidence provided by adding interior parallels, we are willing to infer that parallels do indeed repel rather than attract, which would lead to an anti-Poggendorff effect. Thus, although our data agree with those of Quina and Pollack (1973), who used very similar stimuli consisting of parallel lines and dot pairs, we are unwilling to conclude with them that parallels attract. Furthermore, in the absence of a transversal, directional errors while tracking in the space between parallels were also anti-Poggendorff errors. Clearly, then, since the displays did not contain a transversal, the Poggendorff effect depends upon the presence of a transversal.

\section{EXPERIMENT III}

The dot-setting method used in the first two experiments was again employed with a modified Poggendorff display consisting of a pair of parallel lines with a transversal segment attached to the left parallel only. The modified display has been used before (Weintraub \& Krantz, 1971; Pressey \& Sweeney, 1972; Tong \& Weintraub, 1974). It leads to Poggendorff errors of large magnitude, comparable to those produced by the traditional display containing a transversai segment attached to each parallel, and it is well suited to dot-setting procedures. Administratively, two separate experiments were executed using separate sets of Ss. The tasks required of Ss were different. Stimulus materials were identical.

\section{Method}

The first group of Ss consisted of 40 , the second group of 41 , University of Michigan paid volunteers, who were tested individually. The $S s$ in the first group performed the first two tasks listed below, C and W, and the second group of Ss undertook the remainder. Multilithed in black were lines and dots on white sheets, as described in the first two experiments. Each stimulus display, consisting of parallels and an attached transversal segment, appeared to the left of center on the sheet. Parallels were a pair of lines (always at least twice the length of the transversal), $30 \mathrm{~mm}$ apart. A transversal, $70 \mathrm{~mm}$ in length, was attached to the left parallel at an angle of $221 / 2,45$, or $671 / 2$ deg. Each display was oriented in one of two positions on the sheet: parallel lines upright or transversal upright (see the diagrams in Fig. 6). Included on 
every sheet was a black dot placed randomly, but somewhere near the center of the right half of the sheet.

Procedure. The S sat at a desk covered by a white cloth and placed each sheet in turn in front of himself with the long (11 in.) edge flush with the front edge of the desk. The stimulus displays appeared to $S$ as depicted in Fig. 6 (one to a sheet). He was asked to perform one of several geometric operations.

The free-standing dot to S's right represented the intersection of transversal segment and left parallel. The $S$ was required to place a second dot with respect to the given dot such that attributes of the Poggendorff display, as specified below, were matched in both distance and direction. Repositionings, after erasing the previous judgment, were encouraged. $S$ was requested to circle his final judgment. Symbols for each task listed below correspond to those appearing in Figs. 4-7.

Judge collinearity, $C$ : The task required $S$ to make a Poggendorff judgment with respect to the display, i.e., to estimate a point on the right parallel collinear with the transversal, and then to transfer that estimate to free-standing dots. No mark was permitted on the right parallel. In essence, the requirement was to duplicate a collinearity judgment via a parallelism judgment.

Judge the width between parallels, $W$ : The $S$ positioned a dot at the right so that the free-standing dots duplicated the perceived distance and direction of the width between the parallels measured perpendicular to the parallels at the point of the transversal intersection.

Judge perpendicularity, $\perp$ : From the transversal intersection, $S$ estimated the perpendicular (to the transversal) lying between the parallels, transferring his judgment to free-standing dots. No mark was permitted on the right parallel.

Judge the transversal, $T$ : The $S$ judged the length and orientation of the transversal segment by means of free-standing dots.

Judge collinearity on the display, $[\mathrm{C}]$ : The $S$ marked the point on the right parallel representing the intersection of an imaginery extension of the transversal (the usual Poggendorff task).

Judge perpendicularity on the display [ 1$]$ : From the transversal intersection, $S$ estimated the perpendicular (to the transversal) lying between the parallels and marked its point of intersection on the right parallel itself.

The two preceding tasks, $[C]$, and $[1]$ constitute direct estimates on the display without the encumbrance of a concomitant parallelism judgment. Note that the "direct" assessment procedure cannot be applied to an existing line like the transversal.

\section{Results and Discussion}

The data most pertinent to an explanation of the Poggendorff effect are errors in directionality (slope), so these will be treated first. Figure 4, upright displays, and Fig. 5, tilted displays, present errors in degrees for the various tasks. Conforming with convention, counterclockwise angular errors are designated as positive errors. A plausible and widely held theory concerning the Poggendorff effect is that the transversal orientation is misperceived because acute angles are overestimated (Blakemore, Carpenter, \& Georgeson, 1970; Burns \& Pritchard, 1971). The explanation implies that contour repulsion exists between a transversal and attached parallel such that the transversal would appear tilted too far clockwise (a negative angular error). The transversal, $T$, data do not reveal a consistent negative error. The most striking feature of the transversal data is that the errors are close to zero.

Unlike the orientation of the transversal segment, collinearity (the Poggendorff effect) can be determined not only indirectly by a parallelism

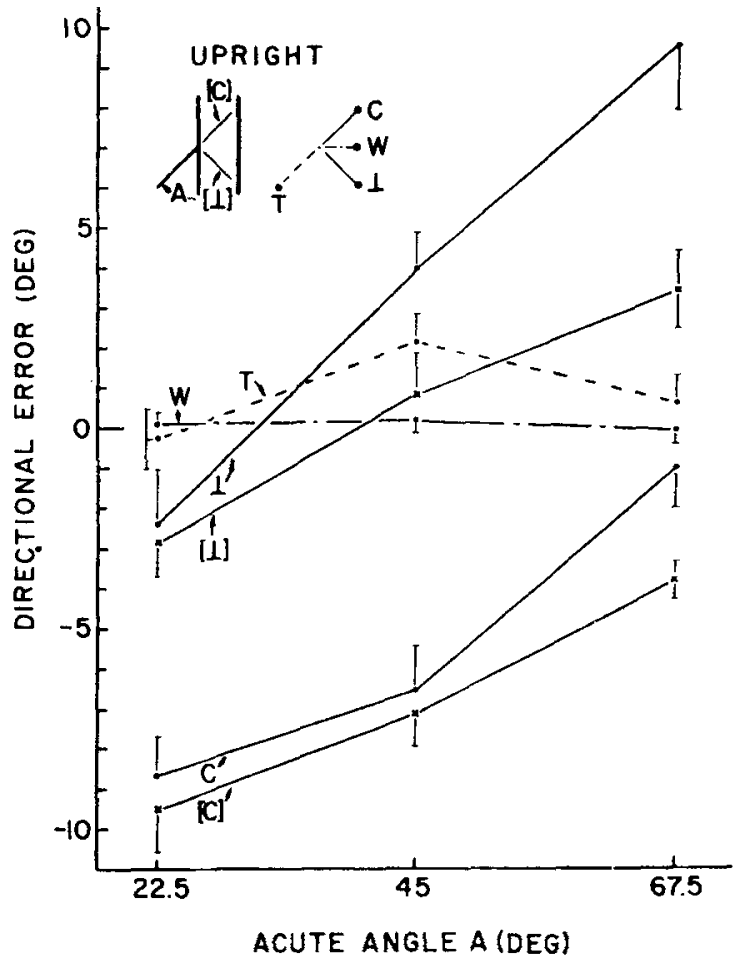

Fig. 4. Mean directional errors, in degrees, for judgments on upright displays in Experiment III. (Counterclockwise errors are designated as positive.)

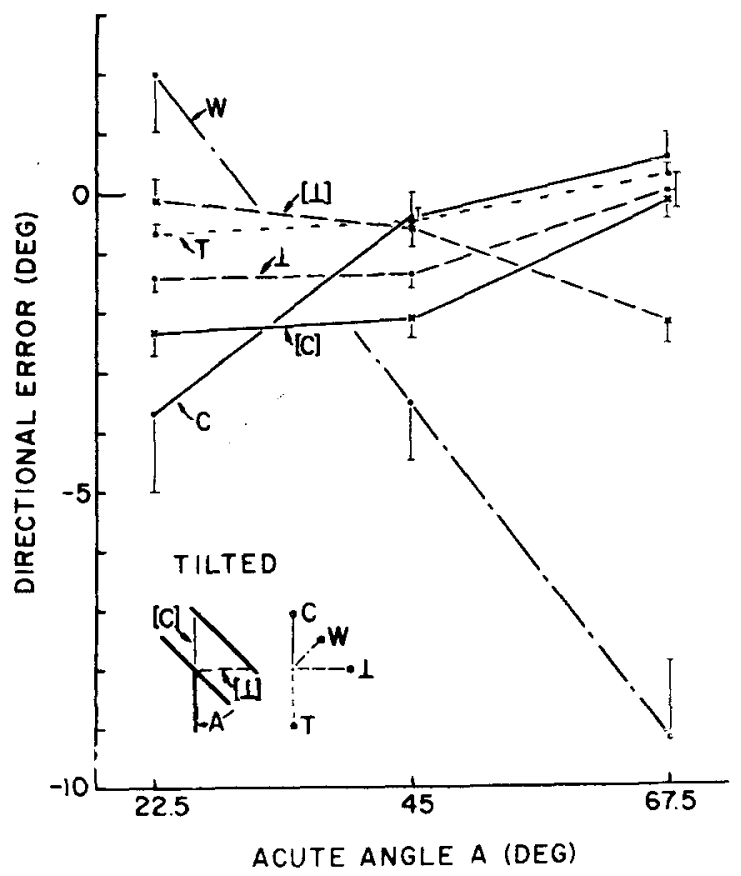

Fig. 5. Mean directional errors, in degrees, for judgments on tilted displays in Experiment III. (Counterclockwise errors are designated as positive.) 
judgment, but also directly on the display. Compare the two types of judgments, $C$ vs $[C]$, in Figs. 4 and 5. An important finding is that, although there are clear differences, the two types of judgment produced similar amounts of error. In addition, collinearity judgments, in contrast to judgments of transversal orientation, produced the expected Poggendorff effect. The Poggendorff effect was expected to be a function of the angle between transversals and parallels. The Weintraub-Krantz (1971) formula for upright displays (the data of Fig. 4) predicts errors for $C$ and $[C]$ of $-3.80,-5.04$, and -3.36 deg for transversal angles of $221 / 2,45$, and $671 / 2 \mathrm{deg}$, respectively. The new data did not confirm the expectation of an error closer to zero at $22 \frac{1}{2} \mathrm{deg}$ than at $45 \mathrm{deg}$, but are otherwise consistent. For any given acute angle between transversal and parallels, the Poggendorff effect was expected to be greatly attenuated when the transversal coincided with the horizontal or vertical axis of the observer's visual field. (See Experiment V of Weintraub \& Krantz, 1971.) Figures 4 and 5 corroborate the expectation, as collinearity errors were always smaller for the same display when tilted. Therefore, collinearity errors measured by two methods generally confirmed expectations with respect to angle subtended between transversal and parallel, and tilt of display.

A general feature of the data can be illustrated by judgments of the width between parallels, W. For an upright display, the free-standing dots were set horizontally without appreciable orientation error $(W$, Fig. 4). For tilted displays, width between parallels is defined along an oblique line and orientation errors were large (W, Fig. 5). The same trends can be seen for collinearity, $C$, and perpendicularity, 1 , judgments. The greatest directional errors occurred with oblique tracking. When judging the orientation of a line physically present, the transversal, $T$, all directional errors were small regardless of the orientation of the transversal. From these findings, and from the similarities between judgments of $\mathrm{C}$ and [C], $\perp$ and [ $\perp$ ], we conclude that the perceptual tasks of determining collinearity and perpendicularity are themselves affected by a change in orientation of the physical stimulus rather than orientation affecting only the perceptual parallelism operation used in transferring a judgment from display to freestanding dots: Appelle (1972) has suggested the term oblique effect, for the pervasive finding that sensitivity declines and veridicality deteriorates for stimuli oriented obliquely.

A new tack in checking the adequacy of Poggendorff theories was to investigate apparent f rpendicularity to determine the correspondence $\mathrm{b}$ ween perpendicularity and collinearity errors. The tasks involved, in one case, imagining an extension of the transversal and, in the other case, imagining a line $90 \mathrm{deg}$ to the transversal. Thus, if the transversal orientation is misperceived and all errors follow as a consequence, then the same angular error should occur in both tasks. With upright displays (Fig. 4), errors for collinearity and perpendicularity were not the same. The noncorrespondence of angular errors constitutes evidence against the hypothesis that the cause of the Poggendorff effect lies in misperceiving the orientation of the transversal.

Judged distances were influenced only modestly by the orientation of the display as shown by the data of Figs. 6 and 7. The size of the angle between transversal and parallel exerted a minor influence on the judged length of $T$ and the distance between parallels, W (Fig. 6). Judged $W$ is significantly less than the objective width between parallels of $30 \mathrm{~mm}$. From the data on upright parallels in Experiment II, it can be calculated that to match a horizontal distance between parallels, free-standing dots should be placed too close together physically by $9.4 \%$ (computed from the value of the $\mathrm{X}$ function for 2 lines in Fig. 3). Under the assumption that the presence or absence of a transversal does not affect judged $W$, applying the correction to the $30-\mathrm{mm}$ width leads to a predicted judged width of $27.2 \mathrm{~mm}$, in excellent agreement with the upright-display data of Fig. 6 . The derivèd Ws shown as dotted lines in Fig. 6 are mean judged widths between the parallels obtained indirectly by computation from judgments of collinearity and perpendicularity. A derived $W$ is not equivalent to a width between parallels judged directly, grounds for rejecting again the hypothesis that the parallels are misperceived in separation and all errors follow accordingly. The widths derived from collinearity estimates are underestimates; the data of Experiment II permit an independent estimate of this error at 45 deg. In Fig. 3, the function of $X$ (from D) at 2 lines shows an underestimation of $15.2 \%$. When applied to a $30-\mathrm{mm}$ width, the prediction is $25.4 \mathrm{~mm}$. This last number is to be compared with $W$ (from collinearity) of an upright display at an acute angle of $45 \mathrm{deg}$ in Fig. 6. The agreement is excellent. We have no explanation to offer for the overestimation shown by the $W$ derived from perpendicularity at $671 / 2$ deg.

The large discrepancies between true and judged distances for collinearity and perpendicularity judgments are plotted in Fig. 7. The graph was designed to emphasize the finding that distance errors were related to the length of the path to be traversed. Judged distances are plotted as a function of the angular deviation of the true track from the shortest possible path-the shortest is the path beginning at the transversal intersection and proceeding perpendicularly across the space between the parallels. All but one distance was underestimated. Any distance estimate associated with a perpendicularity judgment always showed considerably less underestimation than a collinearity track traversing the same objective distance. Much of the error in distance judgments can be accounted for by 


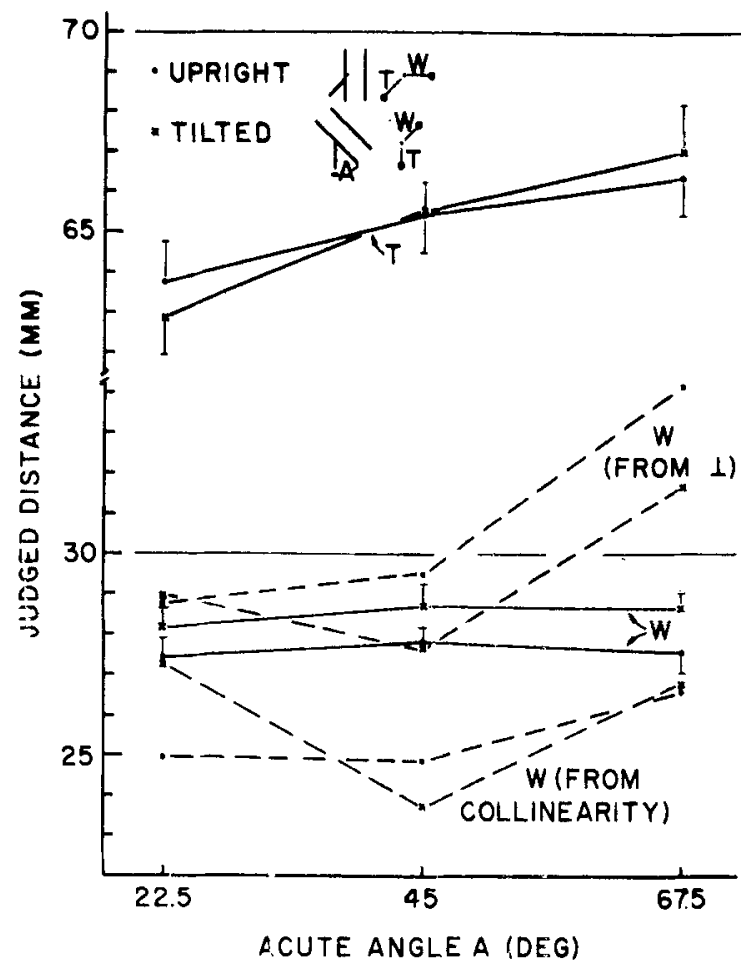

Fig. 6. Mean judgments of transversal length and of width between the parallels in Experiment III. (Derived widths based on perpendicularity and collinearity judgments are also shown.)

applying two assumptions discussed previously. Assume that parallels appear closer together than do free-standing dots. In Fig. 6, for upright displays the underestimation of $W$, averaged across the three acute angles, was $8.1 \%$; for tilted displays, it was $5.0 \%$. Assume, further, that because $\mathrm{S}$ mistracks he therefore intercepts the right parallel too early or too late. In other words, an adjusted "true" distance against which to compare Ss' mean distance judgment is the true distance along the track actually taken by Ss. Using the mean directional error for each condition as an estimate of the track, the adjusted "true" distance can be found by measuring from the start of the track to the intercept of the track with the right parallel. These distances were then corrected by the percentages given in the first assumption. The predictions are given in parentheses next to each plotted mean in Fig. 7. For collinearity judgments, the lower two functions in Fig. 7, the predictions account for a large portion of the underestimated distances. (Even the residual might have been anticipated by noting, from Experiment II, that the 45-deg slant distance between parallels, Fig. 3, showed a greater percent of underestimation than did separation between parallels, W.) When the same corrections are applied to distance associated with perpendicularity judgments, three of the six obtained values are substantially larger than the predicted values (Fig. 7). Again, a problem arises concerning the evaluation of discrepant outcomes. If the objective distance between two free-standing dots lying at a 45-deg angle is the same as that between two free-standing horizontal dots, will the equal slant and horizontal distances appear to be equal? Since such data were not gathered, discrepancies between judged distances made along differently tilted tracks must be interpreted conservatively. It is not possible to determine the influence of the various tilts of the free-standing dots comprising the comparison stimulus.

\section{GENERAL DISCUSSION}

Psychophysical null-match methods, those based upon judgments of equality between stimuli, have high face validity for investigating neurophysiological hypotheses. Judging distance can be cast into the null-match mold: Adjust one distance until it appears equal to another. Parallelism is clearly a null-match operation, judging when one line has the same orientation as another. Collinearity can be interpreted as a special case of parallelism, parallel lines set end to end. What of perpendicularity? When line segments cross, adjacent angles at the intersection can be equated, and, since the angles are supplementary, each will be $90 \mathrm{deg}$. Oftentimes, in the preceding

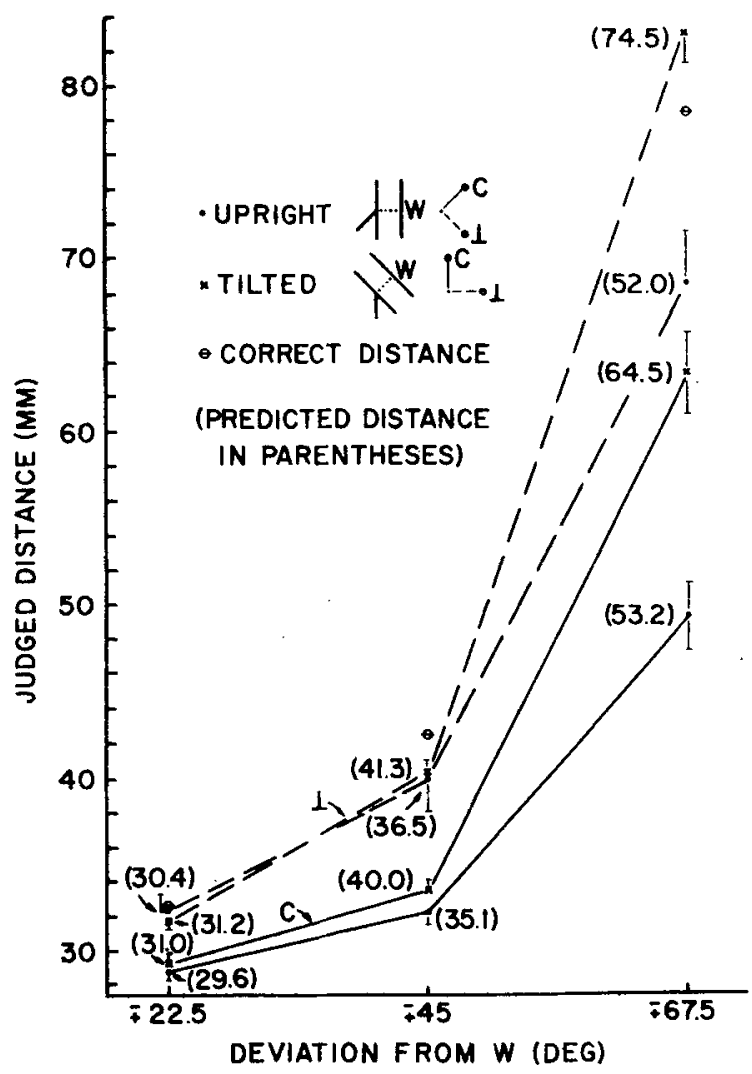

Fig. 7. Mean slant-distance judgments along tracks determined by perpendicularity and collinearity estimates. (Deviation units were used for the abscissa in order to facilitate comparisons among judged lengths based on the same true distance.) 
experiments, more than one null-match operation was required for performing the task.

Brindley (1960) referred to null matches as "Class A" observations, and argued that a simple postulate supported the leap from null-match psychophysics to neurophysiological theory, namely, "... that whenever two stimuli cause physically indistinguishable signals to be sent from the sense organs to the brain, the sensations produced by these stimuli as reported by the subject in words, symbols, or actions must be indistinguishable [p. 144]." Indistinguishability means indistinguishability in all respects. For example, in colorimetry, a metameric (look alike) match is required between a given color and one generated by $S$ from a set of primaries. However, the criterion of indistinguishability in all respects demands a further experimental test: In a two-alternative forced-choice temporal or spatial discrimination experiment, the $S$ must be unable to detect any difference between the look-alike colors. (Compare this treatment of Class A observations with that of Cornsweet \& Pinsker, 1965, and of Sekuler, 1974.)

Unfortunately, complete indistinguishability is impossible in most experiments because the stimuli are indelibly tagged by their contexts. For instance, in an experiment concerning simultaneous color contrast, a stimulus in a chromatic (colored) surround might be matched by a stimulus in an achromatic (neutral) surround. After the color match has been achieved, the stimuli are still distinguishable by their respective surrounds. Since stimuli to be matched are not often context free, of necessity the null matches required are null with respect to selected attributes only. The Poggendorff effect occurs in the presence of parallels, the context tag, while parallels are absent from a comparison stimulus. The $S$ was asked to "disregard" context differences in performing a null match. These less-than-ideal null matches are the best that can be done.

One research strategy is to investigate different null-match operations employing the same Poggendorff display to discover whether the resulting data coincide or whether differences can be resolved by a theory. Requiring that various geometric relationships (distance, collinearity, perpendicularity) be duplicated by a null match for distance and orientation using a pair of free-standing dots proved fruitful. First, it was established that the distance between a pair of parallel lines is underestimated relative to the dots. After allowing for this effect of marking the boundaries of the intervals differently, adding interior lines between parallels gave evidence of a modest Oppel effect, filled space seeming more extensive than unfilled space. From it, one may conclude that if the neurophysiological representations of contours interact, then they repel. Since repulsion will lead to an anti-Poggendorff effect, a descriptive statement of the form, parallels attract, is not an admissible explanation for the Poggendorff effect.

In principle, the experimental technique permits $S$ to judge and set the orientation between a pair of freestanding dots independently of their separation. Such freedom does not exist with the usual judgments made directly on the Poggendorff display, so the causes of misalignment may be hidden. Misperceived orientation of the transversal cannot explain the Poggendorff effect. These errors are small and do not conform to changes in the magnitude of the effect with either a change in the angle subtended between transversal and parallel or a change in the orientation of the entire display. The data support the contention that the primary perceptual anomaly leading to the Poggendorff effect is misperceived collinearity, that is, extending the transversal between the parallels is not performed veridically.

Although both $\mathrm{C}$ and [C] judgments involve perceptual collinearity, the $C$ judgment requires $S$ to perform an additional parallelism operation when transferring the judgment to free-standing dots. The $\mathrm{C}$ and $[\mathrm{C}]$ judgments (and $\perp$ and $[\perp]$ judgments) did not coincide, but they were in reasonable agreement. One may assume that the perceptual collinearity (or perpendicularity) information is not destroyed by the operation transferring it to free dots. Granted that the transversal may be misperceived in orientation, the errors, as assessed by the same parallelism technique employed for Poggendorff collinearity, corresponded in neither direction nor amount to the collinearity errors. The Poggendorff collinearity judgments produced predictably large directional errors; errors in transversal orientation were small. Corroborative evidence comes from Hotopf and Ollerearnshaw $(1972 a, b)$, who obtained a similar finding via the method of constant stimuli, with a free-standing line serving as comparison stimulus. Finally, the anomaly is a collinearity misjudgment associated with the transversal itself, because the perceived orientation of two dots lying obliquely to one another on the parallels in the absence of a transversal showed an anti-Poggendorff effect (Fig. 2, upper function). We therefore feel very confident in rejecting any theory based upon misperceiving the orientation of the transversal. A transversal is necessary, nevertheless.

The data of Experiment III substantiate the influence of two characteristics of Poggendorff displays. The first is the angle subtended between transversal and parallel. The weight of evidence from previous work singles out the obtuse angle as the most critical stimulus feature (Day, 1973; Green \& Hoyle, 1964; Imai, 1973; Krantz \& Weintraub, 1973; Weintraub \& Krantz, 1971). A second critical variable is the orientation of the display in the visual field (Green \& Hoyle, 1964; Leibowitz \& Toffey, 1966; Weintraub \& Krantz, 1971), which conforms to the oblique effect (Appelle, 1972).

Since transversal misorientation is not an 
acceptable explanation, the neurophysiological hypotheses leaning upon contour interactions producing overestimation of acute angles (Blakemore, Carpenter, \& Georgeson, 1970; Burns \& Pritchard, 1971) cannot apply. Versions of Gregory's (1963) misapplied-constancy scaling theory (Gillam, 1971; Green \& Hoyle, 1964) might be modified by postulating that, in order for a parallelism judgment to be feasible for $S$, the free-standing dots are first unconsciously scaled in depth to the same extent as the transversal segment being evaluated. The dot judgments will show no orientation error because both transversal and dots are subject to identical perturbations in depth, and will look equal in orientation when they are physically equal. Although Yarbus (1967, p. 204) has demonstrated a Poggendorff-like eye-tracking error in the presence of a parallel, eye movements are not required to obtain a Poggendorff effect (Pritchard, 1958). The efferentreadiness theory of Festinger, Burnham, Ono, and Bamber (1967), and especially its modifications (Virsu, 1971; Robinson, 1972), are interesting proposals but ones difficult to submit to empirical test.

We suggest that, since the perceptual anomaly involved in the Poggendorff effect is a misjudgment of collinearity, cognitive mistracking is a fitting label for a theory. The label does not deny a possible role for eye movements in the development of Poggendorff errors. But, since eye movements are not necessary to obtain a Poggendorff effect with adults, mistracking is presumed to be a cognitive activity. The label does not deny that contour-interaction processes are at work. Indeed, a critical stimulus feature for a Poggendorff effect is an obtuse angle produced by the actual intersection of transversal and parallel. The label does imply, however, that contour interactions ultimately disturb higher-order stimulus processing. The mistracking interpretation is quite compatible with Pressey's assimilation theory, which he employed to account for the Poggendorff effect (Pressey, 1971; Pressey \& Sweeney, 1972). We cannot bring ourselves to endorse that theory, because it is based upon an imprecisely specified hypothetical construct, the attentive field. Contradictory predictions seem to be obtainable by modifying the locus, size, or shape of the attentive field.

Pinning down the surplus meaning conveyed by the label, cognitive mistracking, is a goal for the future. For the present, we can claim only to have added to the evidence differentiating among broad classes of theory. Our prediction is that, given the diverse independent variables already known to be operating, the ultimate solution to the Poggendorff puzzle will not be captured in an elegant theory grounded on a single principle.

\section{REFERENCES}

Appelle, S. Perception and discrimination as a function of stimulus orientation: The "oblique effect" in man and animals. Psychological Bulletin, 1972, 78, 266-278.

Blakemore, C., Carpenter, R. H. S., \& Georgeson, M. A. Lateral inhibition between orientation detectors in the human visual system. Nature, 1970, 228, 37-39.

Brindley, G. S. Physiology of the retina and the visual pathway. London: Arnold, 1960.

Burns, B. D., \& Pritchard, R. Geometrical illusions and the response of neurons in the cat's visual cortex to angle patterns. Journal of Physiology, 1971, 213, 599-616.

Cornsweet, T. N., \& Pinsker, H. M. Luminance discrimination of brief flashes under various conditions of adaptation. Journal of Physiology, 1965, 176, 294-310.

DAY, R. H. The Poggendorff illusion with obtuse and acute angles. Perception \& Psychophysics, 1973, 14, 590-596.

Festinger, L., Burnham, C. A., Ono, H., \& Bamber, D. Efference and the conscious experience of perception. Journal of Experimental Psychology Monograph, 1967. 74(4, Whole No. 637).

Gillam, B. A depth processing theory of the Poggendortf illusion. Perception \& Psychophysics, 1971, 10, 211-216.

Green, R. T., \& Hoyle, E. M. The influence of spatial orientation on the Poggendorff illusion. Acta Psychologica. 1964, 22, 348-366.

GREGORY, R. L. Distortion of visual space as inappropriate constancy scaling. Nature, 1963, 199, 678-679.

Hotopf, W. H. N., \& Ollerearnshaw, C. The regression to right angles tendency and the Poggendorff illusion. I. British Journal of Psychology, 1972a, 63, 359-367.

Hotopf, W. H. N., \& Ollerearnshaw, C. The regression to right angles tendency and the Poggendorff illusion. Il. British Joumal of Psychology, 1972b, 63, 369-379.

ImaI, S. Experiments on Poggendorff illusion. Journal of Social Sciences \& Humanities (JIMBUN GAKUHO) Tokyo Metropolitan University, 1973, 90, 1-39.

Krantz, D. H., \& Weintraub, D. J. Factors affecting perceived orientation of the Poggendorff transversal. Perception \& Psychophysics, 1973, 14, 511-517.

LEIBowitz, H., \& Toffey, S. The effect of rotation and tilt on the magnitude of the Poggendorff illusion. Vision Research, 1966, 6, 101-103.

Pressey, A. W. An extension of assimilation theory to illusions of size, area and direction. Perception \& Psychophysics, 1971, 9, 172-176.

Pressey, A. W., \& Sweeney, O. Some puzzling results on the Poggendorff illusion. Perception \& Psychophysics, 1972, 12, 433-437.

Pritchard, R. M. Visual illusions viewed as stabilized retinal images. Quarterly Joumal of Experimental Psychology, 1958, 10, 77.81 .

Quini, K., \& Pollack, R. H. Attraction of parallels as a function of intercontour distance. Perceptual \& Motor Skills, 1973, 36, 934 .

Robinson, J. O. The psychology of visual illusion. London: Hutchinson University Library, 1972.

Sekuler, R. Spatial vision. In M. R. Rosenzweig and L. W. Porier (Eds.), Annual review of psychology. Palo Alto: Anrual Reviews, 1974. Pp. 195-232.

Tong, L., \& Weintraub, D. J. Contour displacements and tracking errors. Probing 'twixt Poggendorff parallels. Perception \& Psychophysics, 1974, 15, 258-268.

VIRsu, V. Tendencies to eye movement, and misperception of curvature, direction, and length. Perception \& Psychophysics, $1971,9,65-72$.

Weintraub, D. J., \& Krantz, D. H. The Poggendorff illusion: Amputations, rotations, and other perturbations. Perception \& Psychophysics, 1971, 10, 257-264.

Yarbus, A. L. Eye movements and vision. New York: Plenum Press, 1967. 